\title{
Self-reported perception of healthy eating behaviour through on-line tool is associated with healthy weight status and food intake
}

\author{
R. San-Cristobal ${ }^{1}$, S. Navas-Carretero ${ }^{1,2}$, John C. Mathers ${ }^{3}$ and J.A. Martinez ${ }^{1,2}$ on behalf \\ of Food4Me study \\ ${ }^{1}$ University of Navarra, Centre for Nutrition Research, Department of Nutrition, Food Science and Physiology, \\ Pamplona, Spain, \\ ${ }^{2}$ CIBER Fisiopatología Obesidad y Nutrición (CIBERobn), Instituto de Salud Carlos III, Madrid, Spain and \\ ${ }^{3}$ Human Nutrition Research Center, Institute of Cellular Medicine, Newcastle University, Newcastle Upon Tyne, \\ United Kingdom
}

Prevalence of obesity is still growing in many metropolitan areas, as well as other accompanying chronic diseases, where nutritional status is a relevant health issue. The rise of associated comorbidities related to obesity is prompting government agencies and scientific institutions to develop national policies and guidelines to reduce the increase of body weight in the population. In this sense, websites with a world-wide access appear to be an affordable instrument to contact people, which could be envisaged as a suitable tool in the prevention and the treatment of obesity and comorbidities. For these reasons, the present study aimed to determine the association between on-line self-reported perceptions of eating behaviour and weight status and food intake in the Food4Me cohort.

A sample of 2404 volunteers was selected from individuals who showed interest in the Food4Me study, and signed the informed consent forms to participate in the study through the website (www.food4me.org) in seven European countries. Information about socioeconomic status, medical history, dietary habits and food intake was collected using a questionnaire and participants rated their eating behaviour and health self-perception using Likert scales. Exploratory factor analysis and Cronbach's alpha were used to select items for further analysis. The responses to the selected questions were computed into a score and the results were examined through regression analysis to test associations with reported body mass index and food intake.

From the initial questionnaires, two sets of 8 and 3 self-perception questions (" $8 Q$ " and " $3 Q$ " respectively) were chosen to be analysed. The computed scores presented an inverse association with reported BMI before and after adjustments for age, gender, physical activity level and smoking habits (8Q: $\beta=-0.198, \mathrm{P}<0.001, \mathrm{r}^{2}=0 \cdot 15$; and $3 \mathrm{Q}: \beta=-0 \cdot 266, \mathrm{P}<0 \cdot 001, \mathrm{r}^{2}=0 \cdot 18$ ). Furthermore, the scores were associated with the reported intake of several food groups, the association with fruits and vegetables intake (8Q: $\beta=0.252, \mathrm{P}<0.001, \mathrm{r}^{2}=0.09$; and 3Q: $\left.\beta=0.221, \mathrm{P}<0.001, \mathrm{r}^{2}=0.07\right)$ being the strongest.

The results suggest that the self-perception of eating behaviour, collected by an on-line instrument, may be a useful and feasible component of a screening tool for the determination of nutritional and dietary status in metropolitan population with internet access in order to provide tailored health assessment and precision nutrition.

1. Celis-Morales C, Livingstone KM, Marsaux CF, Macready AL, Fallaize R et al. (2016 Aug 14) Food4Me Study. Effect of personalized nutrition on health-related behaviour change: evidence from the Food4me European randomized controlled trial. Int J Epidemiol. pii: dyw186.

2. Poínhos R, van der Lans IA, Rankin A, Fischer AR, Bunting B, Kuznesof S, Stewart-Knox B, Frewer LJ (2014 Oct 21) Psychological determinants of consumer acceptance of personalised nutrition in 9 European countries. PLoS One 9(10): e110614. doi: 10.1371/journal.pone.0110614. 